\title{
«Vísteme despacio que tengo prisa». Un análisis ético de la vacuna del COVID-19: fabricación, distribución y reticencia
}

\author{
Maite Cruz Piqueras \\ Escuela Andaluza de Salud Pública \\ maiteazul.cruz@gmail.com \\ Joaquín Hortal Carmona \\ Hospital de Guadix, Granada \\ johoca@gmail.com \\ Javier Padilla Bernáldez \\ CS Fuencarral, Madrid \\ javithink@gmail.com
}

Fecha de recepción: 15-6-2020

Fecha de aceptación: 30-6-2020

\section{Resumen}

La llegada de una vacuna frente al SARS-CoV-2 que pueda frenar la expansión de la infección se ha postulado como la respuesta definitiva para frenar la pandemia de COVID-19. Lejos de ser una solución libre de conflictos éticos, la introducción de una nueva vacuna en la práctica de la salud pública, y que se haga en una situación de inestabilidad social, política y económica, vuelve a activar la línea de tensión entre el poder de las instituciones, la autonomía de las personas y las dinámicas de los mercados globales. Esto hace que sea preciso un análisis ético que incluya los conflictos en el ámbito de la investigación y el desarrollo de la vacuna, los elementos de justicia global presentes en la distribución de la vacuna, las decisiones de priorización cuando la oferta de esta no cubra la demanda existente y las posturas que se deberán tomar, desde los poderes públicos, para abordar los casos de reticencia por parte de las personas que no deseen que se les administre la vacuna -o que planteen dudas ante ello-.

Palabras clave: ética de la salud pública; vacunación; reticencia; ética de la investigación; justicia; criterios distribución; COVID-19

Abstract. "Haste makes waste". An ethical analysis of the COVID-19 vaccine: Development, allocation and hesitation

The development of a vaccine against SARS-CoV-2 that can stop the spread of the infection has been postulated as the definitive response to end the COVID-19 pandemic. Far from being free of ethical conflicts, the introduction of a new vaccine in a situation of social, political and economic instability reactivates the tension among institutional power, people's autonomy and the dynamics of global markets. This requires an ethical analysis that includes conflicts in the field of vaccine research and development, glob- 
al justice in vaccine distribution, prioritization decisions when the supply of vaccines does not meet demand and the positions that should be taken by public institutions to address vaccine hesitancy among people who either do not want to take the vaccine or raise doubts about it.

Keywords: public health ethics; vaccination; hesitation; research ethics; justice; allocation; COVID-19

\section{Sumario}

1. Introducción

2. La vacuna: aspectos referidos a ética de la investigación y efectividad 3. Cuestiones de justicia y criterios de distribución. ¿¿A quién poner la vacuna?
4. Reticencia a la vacunación: entre la autonomía y la obligatoriedad

5. A modo de conclusión

Referencias bibliográficas

\section{Introducción}

La pandemia de SARS-CoV-2 que comenzó a expandirse por el mundo en el primer trimestre de 2020 se ha planteado como un problema de salud global. Una de las estrategias para su control definitivo es alcanzar una inmunidad colectiva.

Dado que la inmunidad natural supondría un elevado número de muertes, especialmente entre individuos pertenecientes a colectivos de riesgo (mayores o personas con especial vulnerabilidad clínica), la llegada de una vacuna y la expansión de las políticas de vacunación se plantean como una alternativa deseable para disminuir el impacto de la pandemia sobre la salud y la vida de la población. Pero sin que pequemos de positivismo: con solo 14 vacunas que inmunizan frente a enfermedades víricas y el empeño mundial fallido de cuatro décadas por obtener la del VIH/SIDA, no podemos poner toda la esperanza del fin de la COVID-19 en una vacuna, puesto que no sabemos si habrá, cuándo la habrá ni qué efectividad tendrá.

Todo el mundo sigue con inquietud las investigaciones y el desarrollo de una vacuna para la COVID-19. Una vez que se alcance la fase III (tabla 1) en los ensayos clínicos, surgirán una serie de conflictos éticos sobre los que vale la pena ir reflexionando. Si bien muchos aspectos relacionados con la COVID-19 se han tenido que improvisar, los conflictos éticos que pueden derivarse de la vacuna deben incorporarse a un debate en profundidad. Si algo sabemos de esta pandemia es que para conseguir acciones basadas en la colectividad no solo es necesario ofrecer datos sobre los que poner en marcha medidas, sino también argumentos, razones y, en definitiva, confianza en las instituciones de salud pública (Monrad, 2020).

Ante el eventual desarrollo de una vacuna para hacer frente al SARS-CoV-2 en una situación de pandemia con fuerte impacto en las sociedades a escala 
global, surgen varias cuestiones con un importante componente ético sobre las que se introducen algunas reflexiones: ¿cómo investigar sobre la vacuna con garantías éticas?, ¿cuál es el balance entre riesgos y beneficios en investigación clínica de vacunas en situaciones de pandemia?, ‘cuál debería ser el enfoque de las decisiones justas en un contexto de actuación global?, ¿¿de qué manera se han de establecer las estrategias de priorización?, ¿cuáles son los límites a la reticencia vacunal en este contexto?, ¿se debería obligar a toda la población a vacunarse?

\section{La vacuna: aspectos referidos a ética de la investigación y efectividad}

\subsection{A bordo del Enterprise}

Los días hasta la distribución de la vacuna se cuentan ya en vidas perdidas. Bautizada en EE. UU. como Operación Velocidad Warp (Cohen, 2020b) (velocidad superior a la de la luz teorizada en la serie de ciencia ficción Star Trek), la carrera por la vacuna tiene en el tiempo su principal variable. Mientras voces políticas prometen pocos meses, las vacunas de este siglo han necesitado una media de 10,71 años para su desarrollo, con un mínimo de cinco (Eyal et al., 2020; Pronker et al., 2013).

El mayor tiempo transcurre durante la espera, que puede llegar a ser de años, a que los participantes se infecten de forma natural (fase III, tabla 1). Con el SARS-Cov-2 en libre movimiento, esta exposición natural ya está maximizada, pero difícilmente bajará de doce meses (Eyal et al., 2020).

Los atajos en los tiempos de la ciencia se saldan con malas evidencias que derivan en decisiones de salud pública erróneas (Han et al., 2020; Kramer et al., 2020; London y Kimmelman, 2020). La pandemia de COVID-19 es ejemplo de ello en la proliferación de promesas que se publicitan antes de publicarse (Doroshow et al., 2020), que difícilmente se cumplirán (solo el 6\% de las vacunas en fase preclínica se terminan aprobando).

El atajo hacia la vacuna más discutido durante la pandemia son los llamados Human Challenge Studies (HCS), una forma de acortar la fase III en la que no se espera al contagio natural, sino que se infecta de forma deliberada a los participantes (Eyal, s. f.; Eyal et al., 2020; Jamrozik y Selgelid, 2020). Es muy difícil justificar un daño infligido. Sin embargo, en busca de un bien colectivo tan significativo, los intereses de investigadores, participantes y comunidad se alinean, y ciertas restricciones son soslayables (Chappell y Singer, 2020). Su aceptabilidad pasa por los pasos siguientes: 1) una selección de perfiles del menor riesgo de morbimortalidad (20-29 años sin comorbilidad), y 2) simultáneamente mayor riesgo de infección natural (más de un $50 \%$, p. e. sanitarios expuestos o zonas de alta transmisión); 3) se les garantizaría la mejor asistencia posible con prioridad en situación de recursos escasos, y 4) aislamiento seguro para evitar riesgos a convivientes. En estos casos cobra una especial relevancia un consentimiento informado adecuado que permita una completa comprensión sobre los riesgos, así como la garantía de transparencia y la participación 
Tabla 1. Fases del desarrollo de una vacuna para garantizar su seguridad y eficacia

\begin{tabular}{|c|c|c|}
\hline Fase & Características & $\begin{array}{l}\text { Tiempo } \\
\text { estimado }\end{array}$ \\
\hline \multirow[t]{2}{*}{ Preclínica } & Sobre modelos animales. & \\
\hline & $\begin{array}{l}\text { Una de las vacunas más adelantadas, la de ARN mensajero de la } \\
\text { empresa Moderna, ha soslayado la fase preclínica en base a estu- } \\
\text { dios con vacunas previas (Eyal et al., 2020). }\end{array}$ & \\
\hline Fase I & Sobre un pequeño grupo de unas 100 personas. & 1 año \\
\hline Fase II & Sobre un grupo mayor de 200-500 personas. & 2 años \\
\hline \multirow[t]{2}{*}{ Fase III } & $\begin{array}{l}\text { Estudio multicéntrico con cientos o miles de participantes en varios } \\
\text { países, controlados, aleatorizados y frente a placebo (es el paso } \\
\text { previo a la aprobación). }\end{array}$ & 2 años \\
\hline & $\begin{array}{l}\text { Alternativa: Human Challenge Studies. Estudios con } 25-100 \text { partici- } \\
\text { pantes con bajo riesgo de morbimortalidad y alto riesgo de contagio } \\
\text { a los que se infecta deliberadamente. }\end{array}$ & 1,5 años \\
\hline Fase IV & $\begin{array}{l}\text { Incluye los estudios postaprobación de funcionamiento real de la } \\
\text { vacuna. }\end{array}$ & \\
\hline
\end{tabular}

Fuente: elaboración propia a partir de documento OPS/OMS (2020).

pública en el diseño y desarrollo del estudio (Emanuel et al., 2008; Eyal et al., 2020; Jamrozik y Selgelid, 2020; Plotkin y Caplan, 2020).

Pese al beneficio de obtener la inmunidad con la vacuna o pasar la enfermedad en condiciones óptimas (Eyal, s. f.; Jamrozik y Selgelid, 2020), los HCS se enfrentan a la incertidumbre de los efectos tardíos de la COVID-19 para una ganancia de tiempo de apenas dos o tres meses (Cohen, 2020a; Jamrozik y Selgelid, 2020).

Los límites de la velocidad Warp son los propios de la ciencia: método, rigor, transparencia y confiabilidad.

\subsection{El jardinero infiel}

«Es vergonzoso y horrible escuchar a científicos haciendo este tipo de declaraciones [racistas] en el siglo XXI.» Son palabras del director general de la OMS sobre la propuesta pública de dos médicos franceses de hacer los estudios con la vacuna en África «donde no hay mascarillas ni tratamiento ni reanimación, como se hizo en algunos estudios con el SIDA» (WHO, 2020). Son estudios de doble estándar: investigaciones en países pobres que no serían éticamente aceptables en países ricos, bajo la premisa de que la rama placebo, que no recibe tratamiento, no estaría en desventaja por pertenecer a una población con carencias sanitarias (Hellmann et al., 2020). No hace falta una novela conspirativa de John Le Carré para descubrir que los riesgos de la experimentación suelen recaer en los más desfavorecidos (Monrad, 2020).

El ideal técnico de los estudios es que se realicen en una población que concentre alta probabilidad de contagio. Sin embargo, es probable que esa concentración sea por razones inequitativas: políticas de salud pública débiles y baja protección de profesionales (Jamrozik y Selgelid, 2020). El reclu- 
tamiento de voluntarios para los ensayos de la vacuna debe ser escrupuloso para evitar la explotación de desventajas injustas, tanto en países pobres como entre personas desfavorecidas de los países ricos (Emanuel et al., 2008; Eyal, s. f.).

\subsection{Del laboratorio al mundo}

Que la vacuna se apruebe no significa que se convierta en una buena estrategia de salud pública. Aparte de los factores que evalúan las instituciones de control, hay otros que determinan si la vacuna es una buena herramienta de salud pública. Vacunas como la de la polio o la triple vírica, baratas y que hacen frente a amenazas palpables, fueron fáciles de introducir. Las vacunas de las últimas décadas son más caras y actúan contra patologías no tan comunes ni peligrosas, por lo que se han elaborado modelos de evaluación y priorización basados en los principios de la ética de salud pública (tabla 2) (Verweij, 2017).

Existen razones que justifican la vacunación con márgenes muy amplios de costo-efectividad. Con el número de contagios contados por millones y el ritmo de muertes diarias, con las restricciones y las medidas de confinamiento y las caídas de dos dígitos en la economía global, no hay mucho que debatir

Tabla 2. Principios para la introducción de una nueva vacuna

\begin{tabular}{|c|c|c|}
\hline Field y Caplan (2012) & Thompson et al. (2014) & Verweij (2017) \\
\hline \multirow{11}{*}{$\begin{array}{l}\text { Evidencia. } \\
\text { Riesgos. } \\
\text { Distribución de cargas } \\
\text { en la sociedad. } \\
\text { Probabilidad de que } \\
\text { se logre inmunidad de } \\
\text { grupo o erradicación. } \\
\text { Sopesar con normas } \\
\text { sociales, valores cultu- } \\
\text { rales, etc. } \\
\text { Autonomía, protección } \\
\text { del débil, imperativo } \\
\text { tecnológico. }\end{array}$} & \multirow{5}{*}{$\begin{array}{l}\text { Protección frente al daño. } \\
\text { Evidencia de más alta } \\
\text { calidad. } \\
\text { Advertir sobre seguridad y } \\
\text { eficacia de la vacuna. } \\
\text { Transparencia. }\end{array}$} & \multirow{2}{*}{$\begin{array}{l}\text { Debe dirigirse a enfermedades serias que } \\
\text { sean un problema de salud pública. }\end{array}$} \\
\hline & & \\
\hline & & \multirow{2}{*}{$\begin{array}{l}\text { La enfermedad infecciosa grave indivi- } \\
\text { dualmente y/o con potencial para afectar } \\
\text { a una amplia población. }\end{array}$} \\
\hline & & \\
\hline & & $\begin{array}{l}\text { La vacuna y el propio programa deben } \\
\text { ser efectivos y seguros. }\end{array}$ \\
\hline & $\begin{array}{l}\text { Relación entre el riesgo } \\
\text { y el beneficio aceptable } \\
\text { públicamente. }\end{array}$ & $\begin{array}{l}\text { Los inconvenientes para los participantes } \\
\text { deben ser mínimos. }\end{array}$ \\
\hline & $\begin{array}{l}\text { Las vacunas deben tener } \\
\text { un perfil de seguridad } \\
\text { mayor que otros productos. }\end{array}$ & $\begin{array}{l}\text { La ratio entre riesgo y beneficio debería } \\
\text { ser favorable en comparación con esque- } \\
\text { mas de vacunación u opciones alterna- } \\
\text { tivas. }\end{array}$ \\
\hline & \multirow{4}{*}{$\begin{array}{l}\text { Minimización del estigma. } \\
\text { Obligación moral con las } \\
\text { poblaciones vulnerables. } \\
\text { Confianza pública. }\end{array}$} & El programa de vacunación debería dis- \\
\hline & & \\
\hline & & $\begin{array}{l}\text { La participación debería ser generalmen- } \\
\text { te voluntaria, salvo que la vacunación } \\
\text { obligatoria sea esencial para prevenir un } \\
\text { daño concreto y serio. }\end{array}$ \\
\hline & & $\begin{array}{l}\text { La protección y el fomento de la confianza } \\
\text { pública en el programa de vacunación. }\end{array}$ \\
\hline
\end{tabular}

Fuente: elaboración propia a partir de Field y Caplan (2012), Thompson et al. (2014) y Verweij (2017). 
sobre la magnitud de la amenaza que supone la COVID-19. Pero otra cuestión son los niveles de efectividad de la vacuna que estamos dispuestos a aceptar. No se puede obviar que se administra masivamente a personas sanas, por lo que sus riesgos deben ser muy inferiores a sus beneficios (Verweij, 2017). Con la COVID-19 esta es una aseveración que define un gradiente de aceptabilidad totalmente justificado como estrategia a nivel poblacional para proteger a personas vulnerables, pero no así a nivel individual para quienes tienen un riesgo muy bajo de infectarse y desarrollar una enfermedad grave.

\section{Cuestiones de justicia y criterios de distribución. ¿A quién poner la vacuna?}

En el libro Escalas de justicia (Fraser, 2008), Nancy Fraser juega con la polisemia del término escala para plantear la necesidad de abordar la justicia desde dos planteamientos fundamentales: por un lado, enmarcarla en un orden geopolítico superior al del Estado nación —escala como nivel de amplificación de un mapa-, por otro lado, comprender la justicia como una báscula que trata de equilibrar la distribución de los recursos (o los principios, las capacidades, etc.) - escala como báscula.

Esos dos abordajes resultan pertinentes para pensar en la distribución y en el acceso a la vacuna frente al SARS-CoV-2, representándose tanto en la idea de justicia global que trate de evitar que los accidentes de nacimiento determinen el acceso a la vacuna, como en la elección de criterios de priorización en su distribución. La justicia es, generalmente, un aspecto fundamental en la distribución y el acceso a las vacunas; en el caso de la vacuna frente al SARS$\mathrm{CoV}-2$, el hecho de que toda la población mundial tenga necesidad de ella de forma simultánea hace que la forma en la que se vaya distribuyendo cuando esté disponible sea un aspecto aún más relevante.

\subsection{Justicia global}

La COVID-19 como pandemia enmarca la toma de decisiones en un marco de actuación global. En la toma de decisiones sobre la priorización de unas regiones o países frente a otros en relación con la investigación, la producción y el acceso a la vacuna frente al SARS-CoV-2, subyacen dilemas éticos que no atañen solo a los criterios relacionados con el reparto a nivel individual, sino que también engloban las relaciones de poder entre países, los instrumentos económicos de privatización o el monopolio del conocimiento.

Thomas Pogge afirma lo siguiente:

[...] la cuestión relativa a la justicia distributiva no es la de cómo distribuir una fuente de recursos o la de cómo mejorar una distribución dada, sino la de cómo escoger o diseñar las normas económicas básicas que regulan la propiedad, la cooperación y el intercambio y que, de ese modo, condicionan la producción y la distribución. (Pogge, 2005) 
En base a ello, si queremos asegurar que las medidas en relación con la vacuna del SARS-CoV-2 van a ser justas, deberemos mirar hacia estas dimensiones.

En relación con la propiedad de la vacuna, existen dos aspectos fundamentales que requieren atención por parte de la ética de la salud pública: la titularidad de la innovación vacunal y la existencia o no de monopolios de explotación. Estos dos aspectos están relacionados con los sistemas de patentes que se asocian con limitaciones en el acceso a medicamentos y a innovaciones diagnóstico-terapéuticas, especialmente en países de renta baja y media-baja. La explotación en régimen monopolístico de la comercialización de la vacuna tendría un carácter especialmente conflictivo en virtud de dos aspectos que concurren en el caso de la vacuna frente al SARS-CoV-2: 1) la fuerte presencia de fondos públicos en la investigación, el desarrollo y la generación del marco legislativo favorecedor del proceso de generación de esta vacuna, y 2) el hecho de que se trate de una enfermedad de extensión mundial cuya afectación puede ser mayor en países con unos sistemas sanitarios débiles. Un instrumento administrativo de gestión del conocimiento (la patente) no puede suponer una barrera insalvable para el acceso de una gran parte de la población a una innovación que podría, potencialmente, alargar su vida.

Por otro lado, la COVID-19 ha puesto de manifiesto la interrelación dentro de un mundo globalizado (Meagher et al., 2020). Estrategias frente a la pandemia basadas en evitar o minimizar el lockdown con el fin de mantener activa y competitiva la economía se han mostrado ineficientes cuando los países proveedores o clientes de su economía han tenido que cerrar. Si el sistema social y económico actual se desarrolla en un marco global, con una distribución de cargas y beneficios que suele perjudicar a los denominados perdedores de la globalización, el reparto de los avances científico-técnicos tales como la vacuna frente al SARS-CoV-2 no puede ser en base a la capacidad de compra o a la inversión en $\mathrm{I}+\mathrm{D}$, puesto que la situación económica de unos países guarda una estrecha relación con la situación de otros. Nos enfrentamos a la pandemia de forma colectiva y esto exige solidaridad y reciprocidad, así como un acceso equitativo a los resultados de la investigación, por lo que la escala a la que aplicar estos principios no puede ser la del Estado nación.

Ninguna comunidad, país o continente debería soportar la peor parte de la escasez mundial, por lo que se necesita un liderazgo político planetario que asegure una distribución ética global. Es un imperativo ético pero también un interés compartido: la pandemia nos muestra que los brotes que no se traten en cualquier parte del mundo pueden repercutir en el resto del orbe (Kavanagh et al., 2020).

\subsection{Criterios de priorización}

Como afirma Van Parijs (Parijs y Gonzalo, 1993), el asunto de la justicia puede verse trascendido si se da alguna de las dos condiciones siguientes: 1) situación de abundancia y 2) comportamiento altruista para la distribución de lo existente. En el caso de la vacuna frente al SARS-CoV-2 podemos convenir que, 
al menos en un primer momento, no existirá una situación de abundancia y no se generará en cuestión de pocos meses una oleada de altruismo global que organice el adecuado reparto de los recursos existentes.

Sobre la importancia de que la distribución global atienda a criterios de equidad y no solo de poder de compra o reembolso de la inversión en $\mathrm{I}+\mathrm{D}$ ya hemos hablado en el apartado previo. A continuación nos centramos en la selección de criterios de distribución que ayuden a priorizar la utilización de la vacuna cuando esta esté disponible.

En la tabla 3 se recogen los principios que consideramos especialmente relevantes para la toma de decisiones de priorización en la administración de la vacuna frente al SARS-CoV-2.

Tabla 3. Criterios de priorización

\begin{tabular}{|c|c|}
\hline Criterio & Descripción \\
\hline \multirow[t]{2}{*}{ Valor social } & $\begin{array}{l}\text { Prioridad a las personas que desempeñan labores que han sido con- } \\
\text { sideradas como «esenciales» durante la primera fase de la pandemia: } \\
\text { trabajadores sanitarios; fuerzas y cuerpos de seguridad; cuidadoras de } \\
\text { personas dependientes; personas trabajadoras de la cadena de pro- } \\
\text { ducción, distribución y comercialización de alimentos y bienes básicos; } \\
\text { trabajadores de transportes públicos; etc. }\end{array}$ \\
\hline & $\begin{array}{l}\text { Este criterio no solo es un reconocimiento a la labor desempeñada, } \\
\text { sino que además trataría de garantizar el correcto funcionamiento de } \\
\text { los servicios esenciales en una situación de nueva oleada epidémica. }\end{array}$ \\
\hline
\end{tabular}

Prioridad a las personas Las personas pertenecientes a grupos de riesgo de gravedad clínica de clínicamente más vulnerables

COVID-19 tendrían preferencia en el acceso a la vacuna.

Este criterio trata de proteger de forma preferente a quienes más podrían sufrir el agravamiento de la enfermedad en el caso de contraerla.

Prioridad a las personas Las personas con mayor vulnerabilidad social tendrían prioridad, basánsocialmente más vulne- dose en la existencia de otro tipo de barreras que podrían dificultar su rables acceso normalizado al sistema sanitario en el caso de desarrollar la enfermedad, así como justificado en base a la existencia de condicionantes sociales que supondrían un mayor nivel de exposición al virus y un mayor riesgo de contagio.

Prioridad a embarazadas Reconoce el valor de la gestación siempre que esta tenga posibilidades de llegar a término.

Lotería Criterio igualitario en ausencia de otro criterio superior. Recoge las diversas formas aleatorias de distribución.

Prioridad a quienes ten- De acuerdo con los estudios actuales, esto podría suponer la priorizagan un papel más releción de adolescentes y jóvenes.

vante en las dinámicas de contagio

Quienes desean vacunarse

La voluntad de recibir la vacuna puede ser un criterio de priorización si existe un porcentaje relevante de la población que no la desee, como manera de incrementar el porcentaje de población que la reciba, abordando la reticencia vacunal en una segunda situación de menor escasez de vacunas.

El primero que llegue Disponibilidad de la vacuna en función de su orden de solicitud o compra.

Fuente: elaboración propia a partir de Emanuel et al. (2020) y Melguizo Jiménez et al. (2020). 
La estrategia de priorización que proponemos consistiría en la identificación de dos segmentos de población (un segmento prioritario y otro no prioritario). El segmento prioritario incluiría a aquellas personas que desempeñan una labor esencial en el mantenimiento de la sociedad y en la lucha contra la pandemia (los grupos mencionados en el primer criterio de la tabla), así como a aquellas personas con mayor vulnerabilidad clínica.

En una segunda fase, la expansión de la priorización debería dirigirse hacia personas con mayor exposición por motivos sociales (grupo de individuos con mayor vulnerabilidad social) y personas que podrían desempeñar un papel importante en la diseminación del virus (jóvenes y adolescentes con actividad académica grupal, especialmente).

En una tercera fase, se podría abordar la generalización de la vacuna a toda la población, priorizando a las personas que quieran que se la administren de forma voluntaria y abordando la reticencia dentro de un marco dialógico, tal como se analiza a continuación.

\section{Reticencia a la vacunación: entre la autonomía y la obligatoriedad}

\subsection{Primer saque, empieza el juego}

El 20 de abril de 2020, en plena oleada de la COVID-19 y cuando la vacuna contra el SARS-Cov-2 aún era (y es) una posibilidad tan deseada como remota, el tenista Novak Djokovic escribía lo siguiente en un chat de Facebook: «Personalmente, me opongo a la vacunación y no quisiera que alguien me obligara a vacunarme para poder viajar» (La Vanguardia, 2020).

Supongamos que en este partido Djokovic plantea un juego defensivo en nombre de la autonomía para elegir vacunarse o no, y su contrincante, llamémosle Rafa (de apellido, por ejemplo, Cofiño), contraataca con un juego que representa la colectividad y el bien común. El público, representado por diversos agentes y contextos económicos, políticos y morales, sigue el partido desde la grada. La mayoría de los espectadores permanece en silencio y apoya al jugador español, tan solo algunos sectores amonestan el arbitraje para ensalzar o abuchear las reglas que maneja la jueza de línea llamada Ciencia. El partido se retransmite por la televisión pero se critica que se haga mediante un canal privado que ha comprado los derechos a la televisión pública. Empieza el partido.

\subsection{Se buscan ovejas negras}

Sin haber encontrado una vacuna, ya hay investigaciones en curso que estudian los movimientos de oposición a las mismas y alertan sobre la preocupación por los mensajes que puedan socavar los esfuerzos para, en un futuro, establecer la inmunidad de rebaño al nuevo coronavirus (Megget, 2020). En 2019, hace apenas un año, la Organización Mundial de la Salud identificó la reticencia a la vacunación como una de las diez principales amenazas a la salud 
mundial (OMS, 2019). Aunque esta declaración se realizó tras constatar una creciente disminución en la cobertura de algunas vacunas, también contribuyó a aumentar la confusión entre reticencia y «antivacunas». El movimiento mal llamado antivacunas (puesto que su reivindicación no se fundamenta tanto en el rechazo como en la libertad de la vacunación) representa entre un 1 y un $2 \%$ de la población no vacunada en países de ingresos altos (Draeger et al., 2019). Entre los argumentos que utilizan estos padres y madres para dudar y/o rechazar la vacunación destacan: la no percepción del riesgo, la desconfianza en el paradigma biomédico y la industria farmacéutica, las creencias en un modelo alternativo de salud y la información que reciben de medios muy dispares, entre los que destaca Internet (Díaz Crescitelli et al., 2020). Otra cuestión bien diferente es que un número considerable de brotes de enfermedades no se producen entre "antivacunas", sino entre poblaciones no vacunadas por problemas de accesibilidad al sistema sanitario (Montaño et al., 2015). La causa de las causas que está tras la no vacunación, como ya se está viendo, tendrá también consecuencias visibles en esta pandemia, tanto en el acceso a los tratamientos como a la posible vacuna (Schmidt, 2020).

\subsection{Cuéntame un cuento y verás que contento}

La narrativa de quienes dudan o rechazan la vacunación se vuelve peligrosa cuando la pasamos por alto o la descartamos como fuente de conocimiento (Harman, 2020). Las respuestas de corte exclusivamente utilitarista (aumentar el número de personas vacunadas), estigmatizantes y apoyadas en la presentación de datos y argumentos desde un paradigma biomédico antes que comunitario, se han mostrado insuficientes para responder a este problema (Cruz Piqueras et al., 2019).

Los grupos que se oponen a las vacunas, aún pequeños en tamaño, tienen una estrategia de comunicación muy eficaz y de gran alcance, abundando mensajes de alto contenido emocional. Contrarrestar la difusión de noticias falsas y teorías de la conspiración que abundan en la red, cuestionar las fuentes, solventar estas dudas y hacer partícipe al conjunto de la población de las decisiones que se toman en relación con la fabricación y la distribución de las vacunas propiciará, más que cualquier otra medida, la vacunación (Ball, 2020).

\subsection{De obligaciones morales y jurídicas}

Desde una perspectiva ética, es difícil apoyarse en valores sin tener claros los hechos. En lo que respecta a la vacuna que se descubra, es fundamental conocer cuál será su grado de efectividad. Cuanto más baja sea, mayor cobertura va a necesitar, pero si la efectividad es alta, la reticencia podría ser más tolerable. Aún más, se da la circunstancia en el caso de los menores que la enfermedad suele ser asintomática o leve, por lo que, siendo vectores de contagio, no se benefician significativamente de la misma. Además, ante la incertidumbre de la duración de la inmunidad natural, podría llegar a ser mejor pasar la enfer- 
medad para evitar complicaciones asociadas a una mayor edad. Entonces, ¿qué argumentos morales damos a las personas que no se quieren vacunar o no permiten la vacunación de sus hijos e hijas?

Las vacunas pueden proteger a aquellos sectores de la población que por su vulnerabilidad clínica (con pluripatologías, enfermedades crónicas y personas de mayor edad) y/o social (personas institucionalizadas, personas sin techo, migrantes en situación irregular, personas sin recursos, sin accesibilidad a los servicios sociosanitarios, etc.) necesitan una protección especial. Es precisamente este deber uno de los sustentos morales para sostener la obligatoriedad a la vacunación de la COVID-19.

Por tanto, desde el punto de vista de protección hacia ciertos grupos más vulnerables y el énfasis en la salud de la colectividad no caben muchas dudas: todas las personas tienen la obligación moral (ello no es equivalente a obligación jurídica) de vacunarse. Si se admite una suerte de obligación moral a la vacunación tan solo restaría encontrar un consenso sobre los límites a la libertad personal, ¿hasta dónde se puede llegar a coartar la autonomía de las personas por el bien de la comunidad? En este sentido surgen diferentes propuestas. Las hay que consideran la autonomía desde una perspectiva relacional, por lo que promueven un paternalismo que no solo reste peldaños a la concepción de libertad negativa, sino que también valore la promoción de las capacidades de las personas (Carter et al., 2015), hasta otras que defienden mayor pluralismo moral en la salud pública. Respecto a esta última, la propuesta es la de no subyugar a un solo valor o a una compensación entre dos valores como autonomía y justicia y tener en cuenta otros argumentos de peso que se ven afectados en la decisión de vacunar para impulsar medidas como la educación, la confianza pública, etc. antes que la coacción (Navin y Attwell, 2019).

En cuanto a los datos, hasta el momento existen resultados contradictorios sobre el aumento de coberturas relacionado con políticas de obligatoriedad (ASSET Reports, 2016; Vaz et al., 2020), pero sí hay consenso en que merma la confianza en las políticas públicas. Estamos ante una situación aún más complicada, si cabe, que la propia vacunación (obligatoria o no), como es la recuperación de dicha confianza. La filósofa Onora O’Neill define la confianza como un valor cuyo significado se traduce en "no decir mentiras ni engañar», promover la confianza requiere destruir la mentira, desterrar los bulos y los posibles conflictos éticos que, como se ha visto, está atravesando su fabricación y posterior comercialización.

En consecuencia, la cuestión no radica tanto en la introducción de la obligatoriedad o en su rechazo, sino en valorar la participación del conjunto de actores implicados en la vacunación, así como en informar de modo efectivo y comprensible. La accesibilidad pública a las decisiones que se adoptan en condiciones de transparencia y publicidad, además de una justificación de las mismas y la habilitación de mecanismos de revisión y crítica de las decisiones adoptadas, serían los pasos a seguir en la rendición de cuentas (Daniels, 2000). En caso de imponerse, las medidas deben tener un periodo limitado e ir acompañadas de otras medidas y cumplir unas determinadas condiciones (Dubé et al., 2016). 


\section{A modo de conclusión}

Sin tener una vacuna para hacer frente al SARS-CoV-2 existen multitud de conflictos éticos acerca de su fabricación, sobre los criterios equitativos de distribución y los posibles escenarios, más o menos coactivos, para exigir la cobertura de toda la población. Pero no debemos obviar que las vacunas no son la única medida de salud pública que pueda ponerse en marcha. Sería necesario también limitar el excesivo optimismo en la vacuna como solución técnica y objetiva al problema de la prevención y la salud de las poblaciones, porque obstaculizan otras medidas que pueden ser política y moralmente más controvertidas y producir más equidad (Harrison y Wu, 2020). La ética de la salud pública se ha construido como una tensión entre el poder del Estado y la autonomía de las personas, pero hay que alejarse de significados cercanos a la libertad de elección frente a visiones de tipo relacional que se asienten en el cuidado. En una lógica de cuidado no se trata solo de proporcionar recursos biomédicos y tecnológicos como puede ser la vacuna, sino también los económicos y sociales que permitirían proteger equitativamente al conjunto de la población.

\section{Referencias bibliográficas}

ASSET Reports (2016). Compulsory Vaccination and Rates of Coverage Immunization in Europe. Recuperado el 15 de junio de 2020, de <http://www. asset-scienceinsociety.eu/reports/page1.html>.

BALl, P. (2020). «Anti-vaccine movement could undermine efforts to end coronavirus pandemic, researchers warn». Nature, 581 (7808), 251-251. $<$ https://doi.org/10.1038/d41586-020-01423-4>

Carter, S.M.; Entwistle, V.A. y Little, M. (2015). «Relational conceptions of paternalism: A way to rebut nanny-state accusations and evaluate public health interventions». Public Health, 129 (8), 1021-1029. $<$ https://doi.org/10.1016/j.puhe.2015.03.007>

Chappell, R.Y. y Singer, P. (2020). «Pandemic Ethics: The case for experiments on human volunteers». The Washington Post (27 de abril). Recuperado el 15 de junio de 2020, de <https://www.washingtonpost.com/ opinions/2020/04/27/pandemic-ethics-case-experiments-human-volun teers/?arc $404=$ true $>$.

Cohen, J. (2020a). «Infect volunteers to speed a coronavirus vaccine?». Science, 368 (6486), 16.

$<$ https://doi.org/10.1126/science.368.6486.16>

- (2020b). «U.S. "Warp Speed" vaccine effort comes out of the shadows». Science, 368 (6492), 692-693. <https://doi.org/10.1126/science.368.6492.692>

Cruz Piqueras, M.; Rodríguez García de Cortázar, A.; Hortal Carmona, J. y Padilla Bernáldez, J. (2019). «Reticencia vacunal: Análisis del discurso de madres y padres con rechazo total o parcial a las vacunas». 
Gaceta Sanitaria, 33 (1), 53-59.

<https://doi.org/10.1016/j.gaceta.2017.07.004>

Daniels, N. (2000). «Accountability for reasonableness». BMJ: British Medical Journal, 321 (7272), 1300-1301.

Díaz Crescitelli, M.E.; Ghirotto, L.; Sisson, H.; Sarli, L.; Artioli, G.; Bassi, M.C.; Appicciutoli, G. y Hayter, M. (2020). "A meta-synthesis study of the key elements involved in childhood vaccine hesitancy». Public Health, 180, 38-45.

<https://doi.org/10.1016/j.puhe.2019.10.027>

«Djokovic se opone a vacunarse de la Covid-19 para volver a jugar». La Vanguardia (20 de abril de 2020). Recuperado el 15 de junio de 2020, de <https:/www.lavanguardia.com/deportes/tenis/20200420/48627376597/ djokovic-vacuna-covid-19-coronavirus.html>.

Doroshow, D.; Podolsky, S. y Barr, J. (2020). «Biomedical Research in Times of Emergency: Lessons From History». Annals of Internal Medicine, 173 (4), 297-299. $<$ https://doi.org/10.7326/M20-2076>

Draeger, E.; Bedford, H.E. y Elliman, D.A.C. (2019). «Should measles vaccination be compulsory?». BMJ, 365: 12359. <https://doi.org/10.1136/bmj.12359>

Dubé, E.; Gagnon, D.; Ouakki, M.; Bettinger, J.A.; Guay, M.; Halperin, S.; Wilson, K.; Graham, J.; Witteman, H.O.; MacDonald, S.; Fisher, W.; Monnais, L.; Tran, D.; Gagneur, A.; Guichon, J.; Saini, V.; Heffernan, J.M.; Meyer, S.; Driedger, S.M. y Network, C.I.R. (2016). "Understanding Vaccine Hesitancy in Canada: Results of a Consultation Study by the Canadian Immunization Research Network». PLOS ONE, 11 (6), e0156118. $<$ https://doi.org/10.1371/journal.pone.0156118>

Emanuel, E.J.; Persad, G.; Upshur, R.; Thome, B.; Parker, M.; Glickman, A.; Zhang, C.; Boyle, C.; Smith, M. y Phillips, J.P. (2020). «Fair Allocation of Scarce Medical Resources in the Time of Covid-19». New England Journal of Medicine, 382, 2049-2055. <https://doi.org/10.1056/NEJMsb2005114>

EMANUEL, E.J.; Wendler, D. y GRADY, C. (2008). «An ethical framework for Biomedical Research». En: Emanuel, E.J.; Wendler, D.; Grady, C.; Crouch, R.A.; Reidar, K.L. y Miller, F.G. (eds.). The Oxford Textbook of Clinical Research Ethics. Nueva York: Oxford University Press, 123-135.

Eyal, N. (s. f.). «Why Challenge Trials of SARS-CoV-2 Vaccines Could Be Ethical Despite Risk of Severe Adverse Events». Ethics \& Human Research, 42 (4), 24-34. $<$ https://doi.org/10.1002/eahr.500056>

Eyal, N.; Lipsitch, M. y Smith, P.G. (2020). «Human Challenge Studies to Accelerate Coronavirus Vaccine Licensure». The Journal of Infectious Diseases, 221 (11), 1752-1756 (1 de junio).

<https://doi.org/10.1093/infdis/jiaa152> 
Field, R. y CAPlan, A. (2012). «Evidence-based decision making for vaccines: the need for an ethical foundation». Vaccine, 30 (6), 1009-1013. <https://doi.org/10.1016/J.VACCINE.2011.12.053>

Fraser, N. (2008). Escalas de justicia. Traducido por Antoni Martínez Riu. Barcelona: Herder.

HaN, Z.; WANG, J.; Zhang, K. y TANG, Q. (2020). «The ethics of COVID-19 clinical trials: New considerations in a controversial area». Integrative Medicine Research, 9 (3), 100425. <https://doi.org/10.1016/j.imr.2020.100425>

Harman, S. (2020). «The danger of stories in global health». The Lancet, 395 (10226), 776-777. <https://doi.org/10.1016/S0140-6736(20)30427-X>

HARrison, E.A. y WU, J.W. (2020). «Vaccine confidence in the time of COVID-19». European Journal Epidemiology, 35, 325-330 $<$ https://doi.org/10.1007/s10654-020-00634-3>

Hellmann, F.; Williams-Jones, B. y Garrafa, V. (2020). «COVID-19 and Moral Imperialism in Multinational Clinical Research». Archives of Medical Research, 51 (6), 572-573. $<$ https://doi.org/10.1016/j.arcmed.2020.04.017>

JamroziK, E. y Selgelid, M.J. (2020). «COVID-19 human challenge studies: Ethical issues». The Lancet Infectious Diseases, 20 (8), 198-203. <https://doi.org/10.1016/S1473-3099(20)30438-2>

Kavanagh, M.M.; Erondu, N.A.; Tomori, O.; Dzau, V.J.; Okiro, E.A.; Maleche, A.; Aniebo, I.C.; Rugege, U.; Holmes, C.B. y Gostin, L.O. (2020). «Access to lifesaving medical resources for African countries: COVID-19 testing and response, ethics, and politics». The Lancet, 395 (10238), 1735-1738. <https://doi.org/10.1016/S0140-6736(20)31093-X>

Kramer, J.B.; Brown, D.E. y Kopar, P.K. (2020). «Ethics in the Time of Coronavirus: Recommendations in the COVID-19 Pandemic». Journal of the American College of Surgeons, 230 (6), 1114-1118. <https://doi.org/10.1016/j.jamcollsurg.2020.04.004>

London, A.J. y Kimmelman, J. (2020). «Against pandemic research exceptionalism». Science, 368 (6490), 476-477. $<$ https://doi.org/10.1126/science.abc1731>

Meagher, K.M.; Cummins, N.W.; Bharucha, A.E.; Badley, A.D.; Chlan, L.L. y Wright, R.S. (2020). "COVID-19 Ethics and Research». Mayo Clinic Proceedings, 95 (6), 1119-1123. $<$ https://doi.org/10.1016/j.mayocp.2020.04.019>

MegGET, K. (2020). «Even covid-19 can't kill the anti-vaccination movement». BMJ, 369, m2184.

<https://doi.org/10.1136/bmj.m2184>

Melguizo Jiménez, M.; Hortal Carmona, J. y Padilla Bernáldez, J. (2020). «Medicina basada en las "existencias" o soporte ético para las decisiones difíciles». Actualización en Medicina de Familia, 16 (7). Recuperado de $<$ https://amf-semfyc.com/web/article_ver.php?id=2685>. 
Monrad, J.T. (2020). «Ethical considerations for epidemic vaccine trials». Journal of Medical Ethics, 46 (7), 465-469. <https://doi.org/10.1136/medethics-2020-106235>

Montaño Remacha, C.; Gallardo García, V.; Mochón Ochoa, M.M.; García Fernández, M.; Mayoral Cortés, J.M. y Ruiz Fernández, J. (2015). «Brotes epidémicos de sarampión en Andalucía durante el período 2010-2015». Revista Española de Salud Pública, 89 (4), 407-418. $<$ https://doi.org/10.4321/s1135-57272015000400009>

Navin, M.C. y Attwell, K. (2019). «Vaccine mandates, value pluralism, and policy diversity». Bioethics, 33 (9), 1042-1049. <https://doi.org/10.1111/bioe.12645>

OMS (s. f.). Diez cuestiones de salud que la OMS abordará este año. Recuperado el 24 de junio de 2020, de <https://www.who.int/es/news-room/spotlight/ ten-threats-to-global-health-in-2019>.

OPS/OMS (2020). COVID-19 Fases de desarrollo de una vacuna. Recuperado el 30 de mayo de 2020, de <https://www.paho.org/es/documentos/covid19-fases-desarrollo-vacuna>.

Parijs, P. van y Gonzalo, E. (1993). ¿Qué es una sociedad justa?: Introducción a la práctica de la filosofía política. Barcelona: Ariel.

Plotkin, S.A. y Caplan, A. (2020). «Extraordinary diseases require extraordinary solutions». Vaccine, 38 (24), 3987-3988. $<$ https://doi.org/10.1016/j.vaccine.2020.04.039>

Pogge, T. (2005). La pobreza en el mundo y los derechos humanos. Barcelona: Paidós.

Pronker, E.S.; Weenen, T.C.; Commandeur, H.; Claassen, E.H.J.H.M. y Osterhaus, A.D.M.E. (2013). "Risk in Vaccine Research and Development Quantified». PLoS ONE, 8 (3). $<$ https://doi.org/10.1371/journal.pone.0057755>

Schmidt, H. (2020). «Vaccine Rationing and the Urgency of Social Justice in the Covid-19 Response». The Hastings Center Report, 50 (3), 46-49. <https://doi.org/10.1002/hast.1113>

Thompson, A.; Komparic, A. y SMITH, M.J. (2014). «Ethical considerations in post-market-approval monitoring and regulation of vaccines». Vaccine, 32 (52), 7171-7174. $<$ https://doi.org/10.1016/j.vaccine.2014.10.016>

Vaz, O.M.; Ellingson, M.K.; Weiss, P.; Jenness, S.M.; Bardají, A.; BedNARCZYK, R.A. y OMER, S.B. (2020). «Mandatory Vaccination in Europe». Pediatrics, 145 (2), e20190620. $<$ https://doi.org/10.1542/peds.2019-0620>

VerWEIJ, M.F. (2017). "Ethics of Immunization». Reference Module in Biomedical Sciences, 7-10. <https://doi.org/10.1016/B978-0-12-803678-5.00144-2>

WHO (2020). COVID-19 virtual press conference - 6 April, 2020. Recuperado el 14 de junio de 2020, de <https://www.who.int/docs/default-source/ coronaviruse/transcripts/who-audio-emergencies-coronavirus-pressconference-full-06apr2020-final.pdf?sfvrsn=7753b813_2>. 
Maite Cruz Piqueras es socióloga del área de Gestión de Servicios y Profesionales de la Salud de la Escuela Andaluza de Salud Pública (EASP). Ha participado en la coordinación de diversos proyectos de planificación estratégica y evaluación de programas de salud de la Consejería de Salud y Familias de la Junta de Andalucía, entre los que destacan la Estrategia de Bioética del Sistema Sanitario Público de Andalucía, la puesta en marcha y actualización del Catálogo de Consentimientos Informados, así como el desarrollo de la Red de Comités de Ética Asistencial. En el área de salud global ha sido consultora técnica de proyectos en Uruguay y Chile para el diseño de modelos de redes integradas en Servicios de Salud (RISS). Es directora del Diploma de Especialización en Bioética, título propio de la Universidad de Granada, y docente en varios másteres, diplomas y cursos de ética. En investigación forma parte de la red EOL sobre el final de la vida (End of Life Andalusian Network), la red INEDyTO (Investigación en Ética de la Donación y el Trasplante de Órganos) y ESPACyOS (Ética para la Salud Pública, Acción, Cuidados y Observación Social). Sus publicaciones han derivado de investigaciones en salud con métodos cualitativos, ética de la investigación cualitativa, la utilización de métodos audiovisuales para difusión de resultados. Más recientemente ha profundizado en los discursos que articulan madres y padres sobre la decisión y reticencia a vacunar a sus hijos e hijas. También ha realizado varios vídeos sobre el final de la vida («Al final tú decides», «De muerte somos todos») y otro para promover un diálogo sobre la vacunación \#dialogavacunas. Pertenece a la Comisión de Investigación de la EASP y es vicepresidenta del Comité Institucional de Ética de la EASP. A ratos es @maiteazules.

Maite Cruz Piqueras is a sociologist in Health Services and Health Professionals Management at the Andalusian School of Public Health (EASP). She has participated in the coordination of various strategic planning projects and the evaluation of health programs of the Ministry of Health and Families of the Regional Government of Andalusia, among them the Bioethics Strategy of the Andalusian Public Health System, the implementation and updating of the Catalog of Informed Consents, as well as the development of the Healthcare Ethics Committees Network. In the field of global health, she has served as a technical consultant for projects in Uruguay and Chile for the design of integrated network models in Health Services (RISS). She is the director of the Diploma in Bioethics Specialization of the University of Granada and a teacher in various master's degrees, diplomas, and ethics courses. She is a member of the Andalusian End of Life network (EOL), the Research in Organ Donation and Transplantation Ethics network (INEDyTO) and the Ethics for Public Health, Action, Care and Social Observation network (ESPACyOS). She has published articles on qualitative health research, ethics of qualitative research, and the use of audiovisual methods for the dissemination of results. More recently, her research has focused on parental discourses regarding the decision and reluctance to vaccinate children. She has also produced several videos on the end of life ("At the end you decide", "We are all dead") and another one to promote dialogue on vaccination \#dialogavacunas. She belongs to the EASP Research Commission and is vice-president of the Institutional Committee of EASP ethics. At times she is @maiteazules.

Joaquín Hortal Carmona es licenciado en Medicina (Universidad de Granada), especialista en medicina de familia y comunitaria, y ejerce profesionalmente en las urgencias y hospitalización polivalente del hospital de Guadix (Agencia Sanitaria Pública Hospital de Poniente). También es experto universitario en bioética, vocal del comité de ética asistencial Granada Nordeste, colaborador docente de la Escuela Andaluza de Salud Pública en temas de bioética, ética de salud pública y desmedicalización. Ha realizado investigación en deprescripción, reticencia vacunal (beca Grifols 2015), ética y trasplantes de órganos (proyecto INEDyTO), con especial interés en teorías de justicia y su aplicación en la distribución de órganos y de recursos sanitarios escasos. En el contexto de la pandemia de COVID-19, ha llevado a cabo investigación en criterios de priorización y en actitudes hacia una eventual vacuna. Doctorando en Bioética sobre distribución de órganos para trasplante y miembro de la red ESPACyOS (Ética Salubrista para la Acción, Cuidados y Observación Social). 
Joaquín Hortal Carmona holds a Licentiate of Medicine from the University of Granada. He is a specialist in family and community medicine and practitioner at the emergency and multipurpose hospitalization units of Guadix Hospital of the Public Health Agency of Andalusia. $\mathrm{He}$ also has a postgraduate specialist certificate in bioethics and is member of the Granada Nordeste Healthcare Ethics Committee and the ESPACyOS network on Health Ethics for Action, Care and Social Observation. He is a collaborating teacher at the Andalusian School of Public Health on issues of bioethics, public health ethics and demedicalization. His research lines include deprescription, vaccination reluctance (Grifols grant 2015), ethics and organ transplants (INEDyTO project), with a special focus on theories of justice and their application in the distribution of organs and scarce health resources. In the context of the COVID-19 pandemic, his research focuses on prioritization criteria and attitudes towards a possible vaccine. $\mathrm{He}$ is currently pursuing a PhD in Bioethics on organ distribution for transplantation.

Javier Padilla Bernáldez es licenciado en Medicina (UAM). Es especialista en Medicina Familiar y Comunitaria. Máster en Economía de la Salud y del Medicamento (UPF) y en Salud Pública y Gestión Sanitaria (EASP). Es autor de ¿A quién vamos a dejar morir? (Capitán Swing, 2019) y Epidemiocracia (Capitán Swing, 2020) y co-coordinador de Salubrismo o barbarie (Atrapasueños, 2017). Actualmente trabaja como médico de familia en el Centro de Salud Fuencarral (Madrid). En el ámbito investigador, se ha centrado especialmente en el ámbito de las desigualdades sociales y las teorías de la justicia.

Javier Padilla Bernáldez holds a Licentiate of Medicine from the Autonomous University of Madrid (UAM), a Master of Health and Medicine Economics from Pompeu Fabra University (UPF) and a Master of Public Health and Health Management from the Andalusian School of Public Health (EASP). He is a specialist in family and community medicine and currently works as a family practitioner at the Fuencarral Health Center of Madrid. He is author of ¿A quién vamos a dejar morir? (Capitán Swing, 2019) and Epidemiocracia (Capitán Swing, 2020), and co-coordinator of Salubrismo o barbarie (Atrapasueños, 2017). His primary research interests focus on social inequalities and theories of justice. 